Website: http://revistas.lamolina.edu.pe/index.php/acu/index

(C) Universidad Nacional Agraria La Molina, Lima - Perú

\title{
La cuenca como unidad de gestión para el desarrollo de espacios rurales: un análisis exploratorio
}

\author{
The river basin as a management unit for the development of rural areas: an exploratory analysis
}

\author{
Silvia Rosa Pérez Huamán ${ }^{1}$
}

\begin{abstract}
Resumen
Con el objetivo de evaluar la compatibilidad de la organización territorial distrital en una cuenca hidrográfica para proponerla como un espacio natural para la gestión del desarrollo rural sostenible. Se realizó un análisis de conglomerados, combinando el método no jerárquico de K-medias y el método jerárquico de Ward para 18 distritos y 10 indicadores de desempeño socioeconómico distrital provenientes del Censo de Población y Vivienda 2007, se concluye que los 18 distritos se agrupan en tres conglomerados y estos se distribuyen en la parte baja, media y alta de cada una de las tres subcuencas que pertenecen a la cuenca del río Mala en Cañete-Perú.
\end{abstract}

Palabras clave: Cuenca hidrográfica; análisis de conglomerados; desarrollo rural; desarrollo sostenible.

\begin{abstract}
With the objective of evaluating the compatibility of the district territorial organization in a hydrographic basin to propose it as a natural space for the management of sustainable rural development. A cluster analysis was carried out, combining the non-hierarchical K-means method and Ward's hierarchical method for 18 districts and 10 indicators of district socioeconomic performance from the 2007 Population and Housing Census, concluding that the 18 districts are grouped into three conglomerates and these are distributed in the lower, middle and high part of each of the three sub-basins that belong to the Mala river basin in Cañete-Peru. Keywords: river basin; cluster analysis; rural development; sustainable development.
\end{abstract}

\section{Introducción}

El agua es el articulador natural del ecosistema y de las actividades antropogénicas en el territorio y en su recorrido delimita un espacio natural e idóneo para la gestión del desarrollo rural sostenible: la cuenca hidrográfica, sin embargo, algunos actores tanto públicos como privados e involucrados en la gestión del desarrollo sostienen que las variables de régimen fluvial son insuficientes para explicar el complejo sistema de desarrollo territorial. Por esta razón, hay una persistente tendencia a conducir el desarrollo de espacios rurales con intervenciones asistencialistas y con programas y proyectos de apoyo social que no aprovechan el potencial efectivo endógeno para el desarrollo sostenible.

El enfoque de cuenca ha evolucionado paulatinamente, centrando su atención primeramente en la gestión del recurso hídrico hasta lo que hoy se conoce como cogestión de cuencas, que implica la gestión conjunta y coordinada entre los actores del territorio definido por la divisoria de aguas (Dourojeanni, 1999). La eficacia de la gestión a nivel de cuenca se basa principalmente en el conocimiento de la dinámica de la cuenca y de cada uno de sus componentes así como en el conocimiento, voluntad y capacidad de gestión y participación de los actores que intervienen en la cuenca. Por ello, además de disponer de una base de datos, variables y materiales cartográficos, se requiere de un marco institucional estable y del cual formen parte los actores de la cuenca así como disponer de todos los elementos e instrumentos financieros, legales y políticos (Dourojeanni, 1999).

La experiencia en la implementación de la gestión de cuencas hidrográficas en países como México, Rusia, Colombia, da cuenta de una escasa transparencia de la información sobre los procesos de organización y participación de distintos actores, programas y planes (Perevochychikova \& Arellano-Monterrosas, 2008) que aunada a una visión institucional burocrática y tecnicista de las autoridades de agua no favorece una efectiva participación de actores, y en especial, de las comunidades locales (Buitrago, 2014). Es bajo estas premisas que surge la necesidad de mostrar argumentos objetivos para entender que el enfoque de cuenca puede ser compatible con un enfoque político administrativo distrital para gestionar el desarrollo de un territorio aunque en ambos se usen diferentes escalas o variables para su delimitación.

Con este fin se aplica el análisis de conglomerados jerárquicos y no jerárquicos sobre 18 distritos que conforman la cuenca del río Mala en Cañete, para mostrar que la demarcación distrital y la cuenca hidrográfica pueden tener puntos de confluencia territorial y favorecer la participación de los actores para la gestión del desarrollo territorial a nivel de cuenca. 


\section{Materiales y métodos}

La cuenca de río Mala posee una interesante heterogeneidad que va desde los 25 a casi los 5000 m.s.n.m. y es una cuenca representativa de la ruralidad de la región Lima Provincias. El río Mala toma este nombre después de la confluencia de los ríos Quinches y San Lorenzo, que desde una altura de 1545 m.s.n.m. discurre hacia el litoral peruano. Estos tres ríos dividen a la cuenca del río Mala en tres subcuencas, conformadas por un total de nueve unidades hidrográficas básicas. Su composición desde el punto de vista hídrico, político y espacial se muestra la Tabla 1.

Tabla 1. Unidades hidrográficas de la cuenca del río Mala

\begin{tabular}{|c|c|c|c|}
\hline Subcuencas & $\begin{array}{c}\text { Unidades } \\
\text { hidrográficas }\end{array}$ & Distritos que la componen & $\begin{array}{c}\text { Superficie } \\
\text { en cuenca } \\
(\%)\end{array}$ \\
\hline \multirow{6}{*}{ Mala } & \multirow{6}{*}{ 1. Bajo Mala } & Provincia de Cañete: & \multirow{6}{*}{35} \\
\hline & & Mala, San Antonio, Santa Cruz de Flores, Calango & \\
\hline & & Provincia de Huarochiri: & \\
\hline & & Mariatana & \\
\hline & & Provincia de Yauyos: & \\
\hline & & Quinocay & \\
\hline \multirow{3}{*}{ Quinches } & \multirow[b]{2}{*}{ 2. Quinches } & Provincia de Yauyos: & \multirow[b]{2}{*}{24} \\
\hline & & $\begin{array}{l}\text { Huampará, Ayaviri, Quinches, } \\
\text { Huañec, San Joaquín y Cochas }\end{array}$ & \\
\hline & & Provincia de Huarochiri: & \\
\hline \multirow{7}{*}{ San Lorenzo } & 3. Medio Bajo Mala & Huarochiri, Sangallaya y S. Lorenzo de Quinti & 9 \\
\hline & 4. Tantará & S. Lorenzo de Quinti y Tanta & 6 \\
\hline & 5. Medio Mala & Sangallaya, S. Lorenzo de Quinti y Huarochiri & 3 \\
\hline & 6. Acacache & Sangallaya, S. Juan de Tantaranche y S. Pedro de Huancayre & 10 \\
\hline & 7. Medio Alto Mala & Huarochiri y S. Pedro de Huancayre & 1 \\
\hline & 8. Aguaquiri & Santiago de Anchucaya y S. Pedro de Huancayre & 4 \\
\hline & 9. Alto Mala & Santiago de Anchucaya, Huarochiri y S. Juan de Tantaranche & 8 \\
\hline Total cuenca & & & 100 \\
\hline
\end{tabular}

Fuente: INRENA (2007).

En base a la información distrital del Censo de Población y Vivienda (2007) y la Encuesta Registro Nacional de Municipalidades (RENAMU, 2007) a nivel de distritos, se calcularon los 10 indicadores para los 18 distritos y se procesaron en el programa estadístico informático SPSS. Las pruebas aplicadas fueron la prueba la prueba jerárquica de Ward y la prueba no jerárquica de K-medias. Los 10 indicadores de desempeño socioeconómico distrital que se tomó en cuenta para el análisis de conglomerados (Anexo 1) donde dpob, es la densidad de población distrital expresados en $\mathrm{hab} / \mathrm{km}^{2}$; PEA prim, es la PEA primaria como porcentaje de la PEA total distrital; PBI real pc, es el PBI real per cápita distrital expresado en nuevos soles de 1994; Supcomp, es la superficie per cápita de competencia municipal distrital expresado en $\mathrm{km}^{2}$, que mide el ratio entre la superficie distrital en kilómetros cuadrados y el recurso humano municipal distrital disponible para la gestión local; dcar, es la densidad de red vial distrital y mide la longitud de carretera en kilómetros por superficie distrital en kilómetros cuadrados; ca, es la cobertura de agua potable a nivel distrital expresada como porcentaje del total de viviendas particulares censadas con acceso a agua potable; cl, es la cobertura de luz a nivel distrital expresada como porcentaje del total de viviendas particulares censadas con acceso a luz eléctrica; cd, es la cobertura de desagüe a nivel distrital expresada como porcentaje del total de viviendas particulares censadas que tienen acceso servicio de desagüe; TDE, la tasa de dependencia económica es la carga económica que recae sobre la porción productiva distrital, expresada como el porcentaje que representa la población dependiente (menor de 15 y mayor de 65 años) en la PEA en edad de trabajar (mayor de 15 y menor de 65 años); ESM, la cobertura efectiva de educación secundaria a nivel distrital expresada como el porcentaje de la población total distrital mayores de 15 años que logró como mínimo terminar su educación secundaria.

Análisis de Conglomerado con SPSS para los distritos de la cuenca del río Mala

El análisis de conglomerados es una técnica estadística multivariante que busca agrupar elementos (o variables) tratando de lograr la máxima homogeneidad en cada grupo y la mayor diferencia entre los grupos en base a un conjunto de datos. Al ser una técnica exploratoria, no explica causalidad ni tampoco otorga soluciones únicas ni óptimas, el buen resultado depende del conocimiento y experiencia del investigador.

Existen dos tipos de análisis: los no jerárquicos, que asignan grupos diferenciados que el propio análisis de datos configura, sin que unos dependan de otros y los jerárquicos, que configuran grupos con estructura arborescente, de forma que grupos de niveles más bajos van siendo englobados en otros grupos de niveles superiores La combinación del método jerárquico de Ward con el método no jerárquico de K-medias para los 18 distritos y los 10 indicadores de desempeño socioeconómico distrital implica:

Método de Ward es un método jerárquico asociativo o aglomerativo, que empezará con 18 conglomerados (18 distritos), los cuales se irán agrupando hasta llegar a tener a todos los distritos en un mismo conglomerado. Cuando se unen dos conglomerados, la varianza aumenta, el método Ward une los conglomerados buscando minimizar la varianza dentro de cada conglomerado creando grupos homogéneos y con tamaños similares.

Método de K-medias es un método no jerárquico de reasignación que requiere definir a priori el número de conglomerados deseado (una partición deseada es la formación de tres conglomerados, es decir las tres subcuencas de la cuenca del río Mala) para ir intercambiando los indicadores de desempeño socioeconómico de los conglomerados hasta tener una partición mejor. El algoritmo de partición consistirá en asignar cada indicador de desempeño al conglomerado que se encuentre más próximo del centro, empleando generalmente la distancia euclídea. La distancia euclidiana $\mathrm{d}$ entre dos puntos $\mathrm{P}_{1} \mathrm{y}_{2}$, de coordenadas $\left(\mathrm{x}_{1}, \mathrm{y}_{1}\right)$ y $\left(\mathrm{x}_{2}, \mathrm{y}_{2}\right)$ respectivamente es $\mathrm{d}\left(\mathrm{P}_{1} \mathrm{P}_{2}\right)=$ $\sqrt{(x 2-x 1)^{2}+\left(y^{2}-y_{1}\right)^{2}}$

Ambas pruebas tienen el objetivo de formar grupos 
lo más homogéneo internamente pero a la vez lo más heterogéneo entre ellos. La diferencia radica en que el método jerárquico asume una asociación entre las variables y siempre será posible formar nuevos grupos entre ellos debido a su estructura arborescente. Sin embargo, hay que tener en cuenta que ambas pruebas que no requieren inferencia estadística y como tal, son solo modelos exploratorios.

Ejecutando la prueba de Ward en el programa SPSS, se probaron las diferentes combinaciones y posibilidades de agrupamiento con los 10 indicadores de desempeño socioeconómico distrital, quedando definido cuatro tipos de indicadores que daban el mejor nivel de agrupamiento: la densidad poblacional (dpob), el empleo distrital en el sector primario (PEA prim), la superficie per cápita de competencia municipal y la tasa de dependencia económica (TDE). Una vez definidos los indicadores de desempeño, se obtuvo el cuadro de salida que detalla la formación de los conglomerados de pertenencia (Tabla 2) y un dendograma (Figura 1), que explica gráficamente la estructura arborescente del agrupamiento.

Tabla 2. Conglomerado de pertenencia

\begin{tabular}{llllll}
\hline Caso & 6 & 5 & 4 & 3 & 2 \\
\hline 1:Mala & 1 & 1 & 1 & 1 & 1 \\
2:S. Antonio & 1 & 1 & 1 & 1 & 1 \\
3:S. Cruz F. & 1 & 1 & 1 & 1 & 1 \\
4:Calango & 2 & 2 & 2 & 2 & 2 \\
5:Quinocay & 3 & 3 & 3 & 3 & 2 \\
6:Mariatana & 3 & 3 & 3 & 3 & 2 \\
7:Huampará & 4 & 3 & 3 & 3 & 2 \\
8:Cochas & 4 & 3 & 3 & 3 & 2 \\
9:S. Joaquín & 5 & 4 & 3 & 3 & 2 \\
10:Quinches & 4 & 3 & 3 & 3 & 2 \\
11:Huañec & 6 & 5 & 4 & 2 & 2 \\
12:Ayavirí & 2 & 2 & 2 & 2 & 2 \\
13:SLQ & 2 & 2 & 2 & 2 & 2 \\
14:Sangallaya & 4 & 3 & 3 & 3 & 2 \\
15:SPH & 4 & 3 & 3 & 3 & 2 \\
16:Huarochirí & 4 & 3 & 3 & 3 & 2 \\
17:S. Anchucaya & 6 & 5 & 4 & 2 & 2 \\
18:SJT & 4 & 3 & 3 & 3 & 2 \\
\hline
\end{tabular}

En la Tabla 3 se puede apreciar la conformación de tres conglomerados $(1,2,3)$ : el conglomerado uno formado por los distritos Mala, San Antonio y Santa Cruz de Flores, el conglomerado dos formado por los distritos Calango, Huañec, Ayavirí, San Lorenzo de Quinti y Santiago de Anchucaya y finalmente, el conglomerado tres formado por los distritos de Quinocay, Mariatana, Huampará, Cochas, San Joaquín, Quinches, Sangallaya, San Pedro de Huancayre y San Juan de Tantaranche. A cada conglomerado se le está asignando un color para identificar rápidamente su configuración.

En la Figura 1 se pueda apreciar el dendograma que explica el agrupamiento de manera gráfica y un mapa referencial de localización de los conglomerados en el territorio de la cuenca. Mediante la ayuda gráfica es posible visualizar la distribución espacial de los tres conglomerados en el territorio de la cuenca: conglomerado uno de color amarillo, conglomerado dos de color verde y conglomerado tres de color rosado. También se aprecia la delimitación natural (línea azul) de tres espacios: la subcuenca de Mala, la subcuenca de Quinches y la subcuenca de San Lorenzo.

Por lo tanto, tomando en cuenta el agrupamiento bajo la prueba Ward, es posible organizar los límites políticos administrativos de los distritos en la delimitación hidrográfica de la cuenca, sobre la base de indicadores de desempeño socioeconómico y no del uso de indicadores hidrográficos o fluviales. Entonces, es posible afirmar que la delimitación hidrográfica es compatible con la delimitación territorial distrital. Este hecho, podría favorecer la transición hacia la cuenca como unidad de planeamiento y solo de los recursos hídricos.

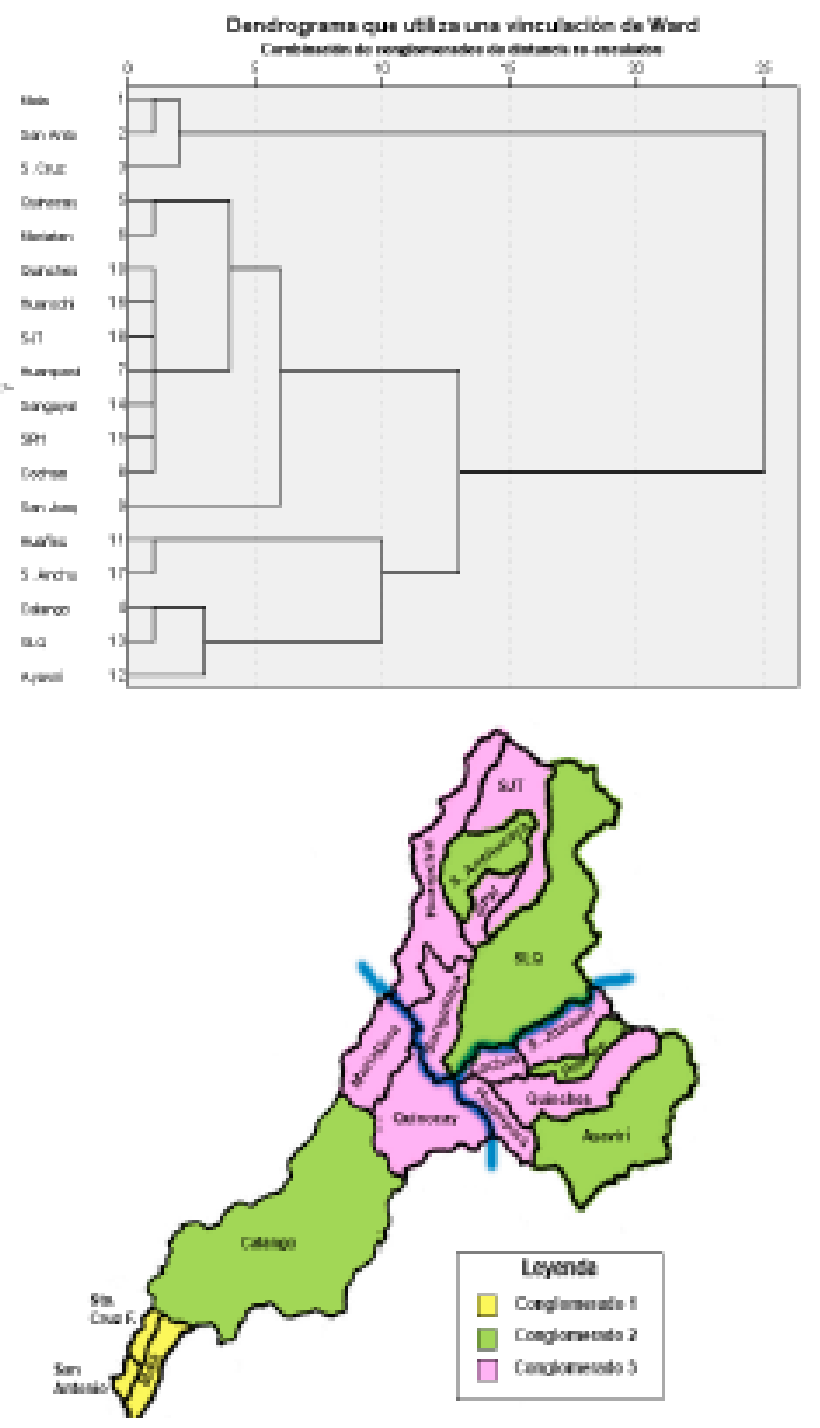

Figura 1. Dendograma de la Prueba de Ward y su representación gráfica en el territorio de la cuenca 
El hecho de poder aproximarse a la cuenca a través de la organización territorial distrital también abre la posibilidad de proponerla como instrumento de operatividad para la regionalización y descentralización a nivel local. La normativa peruana establece en la Ley de Descentralización y Regionalización de 2002 (Ley $\mathrm{N}^{\circ}$ 27783) que "la regionalización se orienta a la constitución de regiones sostenidas, en base al sistema de cuencas y corredores económicos naturales, articulación espacial, infraestructura y servicios básicos, generación efectiva de rentas....". Asimismo, la Autoridad Nacional de Agua ANA elaboró un diagnóstico de cuencas para priorizar las intervenciones a nivel de cuencas, identificando cuencas de prioridad alta, media, baja y muy baja a fin de garantizar el manejo sostenible del recurso hídrico (ANA, 2016). Estos preceptos permiten robustecer la necesidad no solo de trabajar a nivel de cuenca sino también a niveles de cooperación interdistrital. Es decir desde la cuenca hacia los distritos, pero también desde los distritos hacia las cuencas. Asimismo, se procedió a ejecutar la prueba de $\mathrm{K}$-medias, es decir la conformación de tres conglomerados con cuatro indicadores de desempeño socioeconómico distrital antes definidos:

Tabla 3. Centros iniciales de los conglomerados

\begin{tabular}{lccc}
\hline & \multicolumn{3}{c}{ Conglomerado } \\
\cline { 2 - 4 } & 1 & 2 & 3 \\
\hline dpob & 2783,00 & 1171,00 & 2086,00 \\
PEAprim & 77,40 & 19,30 & 67,90 \\
Supcomp & 30,60 & 1,40 & 18,80 \\
TDE &, 95 &, 91 & 1,07 \\
\hline
\end{tabular}

Conglomerado 1: Quinocay, Mariatana, Huampará, San Joaquín, Quinches, Sangallaya, San Pedro de Huancayre, Huarochirií y San Juan de Tantaranche, el cual es semejante al conglomerado tres obtenido de la Tabla 2 (Quinocay, Mariatana, Huampará, San Joaquín, Quinches, Sangallaya, San Pedro de Huancayre, San Juan de Tantaranche y Cochas).

Conglomerado 2:-Mala y San Antonio, el cual es semejante al conglomerado 1 obtenido de la Tabla 2 (Mala, San Antonio y Santa Cruz de Flores).

Conglomerado 3: Calango, Huañec, Ayavirí, San Lorenzo de Quinti y Santiago de Anchucaya el cual es semejante al conglomerado dos obtenido de la Tabla 4 (Calango, Huañec, Huañec, Ayavirí, San Lorenzo de Quinti, Santa Cruz de Flores y Cochas).

Es preciso señalar que ambas pruebas están otorgando similar conformación de grupos relativamente homogéneos. Sin embargo, la prueba Ward otorga una clasificación más compatible con la cuenca, por su característica arborescente. Asimismo, el número de conglomerados se va formado y no se decide a priori como en el caso de la prueba de k-medias.
Por lo tanto, la información de los cuatro indicadores de desempeño socieconómico resultante de la prueba Ward se muestra en la Tabla 5:

Tabla 4. Pertenencia a los conglomerados

\begin{tabular}{cccc}
\hline $\mathrm{N}^{\circ}$ de caso & distrito & Conglomerado & Distancia \\
\hline 1 & Mala & 2 & 30,606 \\
2 & San Antonio & 2 & 30,606 \\
3 & S. Cruz F. & 3 & 289,818 \\
4 & Calango & 3 & 200,467 \\
5 & Quinocay & 1 & 235,978 \\
6 & Mariatana & 1 & 130,115 \\
7 & Huampará & 1 & 62,192 \\
8 & Cochas & 3 & 231,609 \\
9 & San Joaquín & 1 & 100,555 \\
10 & Quinches & 1 & 60,181 \\
11 & Huañec & 3 & 202,946 \\
12 & Ayavirí & 3 & 190,164 \\
13 & SLQ & 3 & 213,111 \\
14 & Sangallaya & 1 & 57,075 \\
15 & SPH & 1 & 25,682 \\
16 & Huarochirí & 1 & 200,954 \\
17 & S. Anchucaya & 3 & 45,857 \\
18 & SJT & 1 & 161,776 \\
\hline
\end{tabular}

Tabla 5. Indicadores de desempeño socioeconómico distrital y la conformación final

\begin{tabular}{|c|c|c|c|c|c|c|}
\hline \multirow[b]{2}{*}{ Conglomerado } & \multirow[b]{2}{*}{ Distritos } & \multirow{2}{*}{$\begin{array}{c}\text { Altura } \\
\text { m.s.n.m. }\end{array}$} & \multirow{2}{*}{$\begin{array}{c}\text { dpob } \\
\text { hab/km }{ }^{2}\end{array}$} & \multicolumn{2}{|c|}{ PEA prim Supcomp } & \multirow{2}{*}{$\begin{array}{c}\text { TDE } \\
\% \text { pea } t\end{array}$} \\
\hline & & & & $\%$ PEA t & $\mathrm{Km}^{2} \mathrm{pc}$ & \\
\hline \multirow{3}{*}{1} & Mala & 30 & 216 & 37.6 & 1.5 & 90.3 \\
\hline & San Antonio & 36 & 98 & 49.8 & 1.4 & 90.8 \\
\hline & S. Cruz F. & 85 & 25 & 56.0 & 7.1 & 77.2 \\
\hline \multirow{5}{*}{2} & Calango & 305 & 4 & 85.9 & 88.5 & 81.6 \\
\hline & Ayavirí & 3235 & 3 & 79.3 & 26.5 & 109.2 \\
\hline & Huañec & 3202 & 12 & 58.7 & 6.3 & 119.3 \\
\hline & SLQ & 2680 & 4 & 79.2 & 66.8 & 91.4 \\
\hline & S. Anchucaya & 3384 & 6 & 78.6 & 13.4 & 106.7 \\
\hline \multirow{10}{*}{3} & Quinocay & 2652 & 4 & 87.4 & 30.6 & 74.5 \\
\hline & Mariatana & 3534 & 8 & 79.3 & 24.1 & 95.3 \\
\hline & Huampará & 2478 & 5 & 78.7 & 10.8 & 89.0 \\
\hline & Cochas & 2831 & 11 & 77.6 & 4.0 & 90.6 \\
\hline & S. Joaquín & 2947 & 8 & 62.9 & 5.9 & 57.7 \\
\hline & Quinches & 2962 & 9 & 78.9 & 37.8 & 88.0 \\
\hline & Sangayalla & 2738 & 8 & 86.7 & 10.2 & 81.5 \\
\hline & SPH & 3135 & 6 & 81.1 & 8.4 & 80.6 \\
\hline & Huarochirí & 3146 & 6 & 64.1 & 16.6 & 88.6 \\
\hline & SJT & 3436 & 4 & 73.5 & 27.4 & 85.3 \\
\hline
\end{tabular}

Fuente: Censo de Población y Vivienda 2007, RENAMU 2007

Con la información organizada se aprecia que el primer conglomerado tiene una conformación muy notoria puesto que se organizan y se agregan los distritos con mayor densidad poblacional. Mientras la densidad poblacional baja, aumenta la PEA primaria (agricultura principalmente) y la superficie per cápita de la competencia municipal e inversamente ocurre con la tasa de dependencia económica. Este resultado de agrupamiento se explica porque estos tres distritos son el centro económico comercial, concentran también servicios de educación y hay una alta tasa de migración de las cuencas altas hacia la parte baja, razón por la cual aumenta la tasa de dependencia económica. 
En el segundo conglomerado, las densidades de población son menores y aumenta la PEA primaria, la superficie per cápita de competencia municipal y la tasa de dependencia económica. Este conglomerado se ubica a mayor altura, hay una mayor presencia de productores agropecuarios y la presencia de población juvenil que labora en el agro aumenta la tasa de dependencia económica.

El tercer conglomerado es muy similar pero está a menor altura, por lo que hay mayores facilidades de acceder a los servicios de la parte baja, tanto para educación como por razones laborales, es por eso que la tasa de dependencia económica baja pero ligeramente.

De lo anterior es posible concluir que los tres conglomerados responden a una dinámica en función a la densidad población y las limitantes de la altura, las cuales ponen restricciones al desarrollo económico del territorio. La altura también está asociada a los pisos ecológicos de la cuenca baja, media y alta, la cual también se aprecia cuando se transita a nivel de las tres subcuencas.

\section{Conclusiones}

Con el análisis de conglomerado y utilizando las pruebas de Ward y de K-medias es posible obtener agrupamientos que están relacionados a la organización territorial y de cuencas, por lo tanto con ambos métodos se ha encontrado la compatibilidad esperada.

El método de Ward y su clasificación final otorga la formación de grupos idóneos, ya que los indicadores de desempeño socioeconómicos distrital están muy relacionados y además la misma estructura arborescente del método es compatible con la organización de cuencas en varios niveles (subcuencas, microcuencas, macrocuencas, etc.)

La conformación territorial distrital es compatible a la organización a nivel de cuenca, debido a que los 3 conglomerados se distribuyen a lo largo del territorio de la cuenca y se logra que en cada subcuenca exista la diversidad de los tres conglomerados asociados a las características de la parte baja, media y alta de una cuenca Así, en la subcuenca de Mala están presentes 3 distritos del conglomerado 1 (Mala, San Antonio y Santa Cruz de Flores), 1 distrito del conglomerado 2 (Calango) y 2 distritos del conglomerado 3 (Mariatana y Quinocay). La subcuenca de Quinches comprende 2 distritos del conglomerado 2 (Ayavirí y Huañec) y 4 distritos del conglomerado 3 (Huampará, Cochas, San Joaquín y Quinches). La subcuenca de San Lorenzo comprende 2 distritos del conglomerado 2 (San Lorenzo de Quinti y Santiago de Anchucaya) y 4 distritos del conglomerado 3

(Sangallaya, Huarochirí, San Pedro de Huancayre y San Juan de Tantaranche). Esta conformación es compatible a la distribución territorial de la cuenca detallada en la Tabla 1. Entonces, partiendo de los distritos y de los indicadores de desempeño socieconómico se llegó a una organización similar a la cuenca sin usar variables hídricas o fluviales.
Por lo tanto, aunque la cuenca y el distrito posean diferentes escalas de medición, el agrupamiento de distritos hace posible una compatibilidad entre ellos. En ese sentido, es posible aproximarnos a la cuenca a través el agrupamiento de los distritos y fomentar espacios de cooperación interdistrital para el manejo sostenible del Recurso hídrico y para consensuar intervenciones en la cuenca para el desarrollo sostenible de los espacios rurales.

\section{Literatura citada}

ANA [Autoridad Nacional del Agua]. 2016. Priorización de cuencas para la gestión de recursos hídricos. Lima, Perú. Disponible en: Website: www.ana.gob.pe

Buitrago, O. 2014. La gestión de cuencas hidrográficas en Colombia y su papel en la solución de conflictos por el uso del agua. Caderno Prudentino de Geografia, Presidente Prudente, 36 (Volumen Especial): 106-125.

Censo de Población y Vivienda. 2007. Sistema Estadístico Nacional del país, ejecutó el 21 de octubre del 2007, los Censos Nacionales: XI de. Población y VI de Vivienda Disponible en: http://censos.inei.gob.pe/cpv2007/ tabulados/.

Dourojeanni, A. 1999. Gestión de cuencas y ríos vinculados con centros urbanos. CEPAL, División de Recursos Naturales e Infraestructura.

Dourojeanni, A. 1994. Políticas públicas para el Desarrollo Sustentable: La gestión integrada de cuencas. CEPAL, División de Recursos Naturales e Infraestructura.

INRENA . 2007. Disponible en: http://sinia.minam. gob.pe/fuente-informacion/instituto-nacionalrecursosnaturales-inrena.

LEY N ${ }^{\circ}$ 27783. 208. Ley de Bases de la Descentralización. Disponible en: http://www2.congreso.gob.pe/sicr/ cendocbib/con4_uibd.nsf/8B3C23D0EB9643D605257 FD400782856/\$FILE/27783.pdf

Perevochtchikova, M. y Arellano-Monterrosas, J. 2008. Gestión de cuencas hidrográficas: experiencias y desafíos en México y Rusia. En: Revista Latinoamericana de Recursos Naturales, 4 (3): 313325.

Encuesta Registro Nacional de Municipalidades -RENAMU. 2007. Disponible en: https://webinei.inei. gob.pe/anda_inei/index.php/catalog/380 
Anexo 1. Los indicadores de desempeño socioeconómico distrital utilizados en el análisis de conglomerados

\begin{tabular}{|c|c|c|c|c|c|c|c|c|c|c|c|}
\hline Distritos & $\begin{array}{c}\text { Altura } \\
\text { m.s.n.m. }\end{array}$ & $\begin{array}{c}\text { dpob } \\
\text { hab } / \mathrm{km}^{2}\end{array}$ & $\begin{array}{l}\text { PEA prim } \\
\% \text { PEA t }\end{array}$ & $\begin{array}{c}\text { PBI real pc } \\
\text { NS/hab }\end{array}$ & $\begin{array}{c}\text { Supcomp } \\
\mathrm{Km}^{2} \mathrm{pc}\end{array}$ & $\begin{array}{c}\text { dcar } \\
\mathrm{km} / \mathrm{km}^{2}\end{array}$ & $\begin{array}{c}\text { ca } \\
\% \text { Viv. }\end{array}$ & $\begin{array}{c}\text { cl } \\
\% \text { Viv. }\end{array}$ & $\begin{array}{c}\text { cd } \\
\% \text { Viv. }\end{array}$ & $\begin{array}{c}\text { TDE } \\
\% \text { pea } t\end{array}$ & $\begin{array}{c}\text { ESM } \\
\%\end{array}$ \\
\hline Mala & 30 & 216 & 37.6 & 3856 & 1.5 & 0.2949 & 61.6 & 79.4 & 51.3 & 90.3 & 77.6 \\
\hline San Antonio & 36 & 98 & 49.8 & 3665 & 1.4 & 0.6076 & 64.8 & 71.1 & 56.2 & 90.8 & 80.2 \\
\hline S. Cruz F. & 85 & 25 & 56.0 & 5497 & 7.1 & 0.1848 & 60.0 & 65.3 & 44.1 & 77.2 & 75.1 \\
\hline Calango & 305 & 4 & 85.9 & 6997 & 88.5 & 0.0809 & 60.2 & 59.0 & 25.2 & 81.6 & 64.4 \\
\hline Quinocay & 2652 & 4 & 87.4 & 8711 & 30.6 & 0.1184 & 63.4 & 63.9 & 14.2 & 74.5 & 64.8 \\
\hline Mariatana & 3534 & 8 & 79.3 & 7574 & 24.1 & 0.0793 & 0.0 & 14.9 & 0.9 & 95.3 & 29.3 \\
\hline Huampará & 2478 & 5 & 78.7 & 8166 & 10.8 & 0.1601 & 84.1 & 82.2 & 57.0 & 89.0 & 64.9 \\
\hline Cochas & 2831 & 11 & 77.6 & 7114 & 4.0 & 0.6643 & 73.8 & 62.1 & 6.8 & 90.6 & 63.2 \\
\hline S. Joaquín & 2947 & 8 & 62.9 & 8283 & 5.9 & 0.0582 & 71.3 & 63.5 & 46.1 & 57.7 & 71.0 \\
\hline Quinches & 2962 & 9 & 78.9 & 8159 & 37.8 & 0.1048 & 68.3 & 80.6 & 21.9 & 88.0 & 57.5 \\
\hline Huañec & 3202 & 12 & 58.7 & 5769 & 6.3 & 0.0714 & 78.6 & 82.3 & 40.6 & 119.3 & 62.3 \\
\hline Ayavirí & 3235 & 3 & 79.3 & 5807 & 26.5 & 0.1125 & 85.5 & 83.3 & 38.3 & 109.2 & 61.4 \\
\hline SLQ & 2680 & 4 & 79.2 & 7057 & 66.8 & 0.0488 & 75.3 & 72.5 & 43.5 & 91.4 & 56.5 \\
\hline Sangayalla & 2738 & 8 & 86.7 & 8152 & 10.2 & 0.2494 & 1.6 & 58.9 & 0.8 & 81.5 & 45.3 \\
\hline SPH & 3135 & 6 & 81.1 & 7909 & 8.4 & 0.1092 & 0.0 & 84.4 & 34.4 & 80.6 & 57.6 \\
\hline Huarochirí & 3146 & 6 & 64.1 & 7346 & 16.6 & 0.1011 & 5.1 & 76.9 & 43.5 & 88.6 & 61.9 \\
\hline S. Anchucaya & 3384 & 6 & 78.6 & 6528 & 13.4 & 0.0123 & 77.0 & 82.5 & 15.3 & 106.7 & 59.8 \\
\hline SJT & 3436 & 4 & 73.5 & 7469 & 27.4 & 0.0648 & 78.8 & 66.7 & 5.8 & 85.3 & 63.0 \\
\hline
\end{tabular}

Fuente: Censo de Población y Vivienda 2007, RENAMU 2007 